\title{
A DIFFERENCE PROPERTY FOR POLYNOMIALS AND EXPONENTIAL POLYNOMIALS ON ABELIAN LOCALLY COMPACT GROUPS $\left({ }^{1}\right)$
}

\author{
BY \\ F. W. CARROLL
}

1. Introduction. Let $f$ be a complex-valued function on $E^{m}$, Euclidean $m$-space, which has the property that for each $h \in E^{m}$, the function $\Delta_{h} f$ : $\Delta_{h} f(x)=f(x+h)-f(x)$ is continuous (or is a polynomial, or an exponential polynomial). Then $f$ itself need not be continuous (or a polynomial, or an exponential polynomial), for there exist nonmeasurable additive functions on $E^{m}$, that is, nonmeasurable solutions $\Gamma$ of the functional equation $\Gamma(x+y)$ $=\Gamma(x)+\Gamma(y)$. However, de Bruijn [1], [2] (for $E^{1}$ ) and Kemperman [7], [8] (for $E^{m}$ ) showed that, among many others, the classes of continuous functions, polynomials, and certain classes of trigonometric and exponential polynomials have the property that if $\Delta_{h} f$ is in the class for each $h \in E^{m}$, then there exists an additive function $\Gamma$ on $E^{m}$ such that $f-\Gamma$ is in the class.

Let $G$ be an abelian topological group, and let $\Omega$ be a class of complexvalued functions on $G$ which contains the constant functions, and such that $f, g \in \Omega$ implies $f-g \in \Omega$ and $f_{h} \in \Omega$ for each $h \in G$, where $f_{h}(x)=f(x+h)$. The class $\Omega$ is said to have the difference property if the following implication holds: let $f$ be a complex-valued function on $G$ such that $\Delta_{h} f \in \Omega$ for each $h \in G$. Then there is an additive function $\Gamma$ on $G$ such that $f-\Gamma \in \Omega$.

Except where the contarary is explicitly stated, $G$ will denote an abelian locally compact group. The product of $m$ copies of the reals will be denoted by $E^{m}$, and the group of integers by $C$. All functions considered are complexvalued. It is known [3] that the class of continuous functions on $G$ has the difference property. The principal results of this paper are Theorems 1 and 2 , which give necessary and sufficient conditions on $G$ in order that the class of polynomials on $G$ (as defined, for instance, in [6]) and the class of exponential polynomials on $G$ have the difference property.

2. The difference property for polynomials. $A$ function $P$ on $G$ is a polynomial of degree $N(N<\infty)$, provided

(P1) for each $(a, b) \in G \times G$, the function

$$
P_{a b}: P_{a b}(\lambda)=P(a+\lambda b) \quad(\lambda \in C)
$$

is a polynomial on $C$.

Presented to the Society, January 28, 1960; received by the editors May 4, 1962, and, in revised form, August 16, 1963.

( ${ }^{1}$ ) This research is a revision of a portion of the author's doctoral dissertation at Purdue University. 
(P2) if $N_{a, b}$ denotes the degree of $P_{a, b}$, then $N=\max \left\{N_{a, b}:(a, b) \in G \times G\right\}$, and

(P3) $P$ is continuous on $G$.

An element $b \in G$ is said to be compact if the closure of the subgroup generated by $b$ is compact. The set $B$ of all compact elements is a closed subgroup [11]. Since $G=E^{m}+G^{\prime}$, where $G^{\prime}$ contains a compact open subgroup [12], it follows that $G / B=E^{m}+G_{1}$, where $G_{1}$ is discrete. If a function $P$ has properties (P1) and (P3) on $G$, if $a \in G$ and $b \in B$, then the set of polynomial values $\left\{P_{a b}(\lambda): \lambda \in C\right\}$ is bounded, since $\{a+\lambda b: \lambda \in C\}$ has compact closure. Thus $P_{a b}(\lambda) \equiv P(a)$, so that $P$ is constant on cosets of $B$, and is essentially a function on $G / B$.

A set $H \subset G$ is a Hamel basis for $G$ provided that for no finite subset $\left\{b_{0}, b_{1}, \cdots, b_{k}\right\}$ of $H$ do there exist integers $N \neq 0, n_{1}, \cdots, n_{k}$ such that $N b_{0}$ $=n_{1} b_{1}+\cdots+n_{k} b_{k}$, and provided that $H$ is maximal with respect to this property. It follows from the Hausdorff maximal principle that every abelian group has a (possibly empty) Hamel basis. If $x \in G$, then there exist uniquely determined elements $b_{1}, \cdots, b_{k}$ in $H$ and integers $N \neq 0, n_{1}, \cdots, n_{k}$ such that

$$
N x=n_{1} b_{1}+\cdots+n_{k} b_{k} \text {. }
$$

If (2.1) holds, we shall write

$$
x \sim\left(n_{1} / N\right) b_{1}+\cdots+\left(n_{k} / N\right) b_{k}=r_{1}(x) b_{1}+\cdots+r_{k}(x) b_{k} .
$$

In this way there is associated with each $b_{\alpha} \in H$ a rational-valued function $r_{\alpha}$ on $G$, and $r_{\alpha}$ is easily seen to be additive.

THEOREM 1. Let $G$ be an abelian locally compact group, and let $B$ be the group of compact elements of $G$. A necessary and sufficient condition in order that the class of polynomials on $G$ have the difference property is that $G / B=E^{m}+G_{1}$, where $G_{1}$ has a finite Hamel basis.

The following example shows the necessity of the condition.

Example 1. Let $G / B=G_{1}+E^{m}$ where $G_{1}$ is discrete and has an infinite Hamel basis. Then there exists a continuous function on $G$ which is not a polynomial, but each of whose differences is a polynomial. It suffices to show that such a function $f$ exists on $G_{1}$, for then the function

$$
f_{1}: f_{1}(x)=f\left(P_{2} P_{1} x\right),
$$

where $P_{1}$ and $P_{2}$ are the natural mappings from $G$ to $G / B$ and from $G / B$ to $G_{1}$ respectively, is an extension of $f$ which has the required properties on $G$. Let $b_{1}, b_{2}, \ldots$ be a countably infinite subset of the Hamel basis of $G_{1}$, let $r_{1}, r_{2}, \ldots$ be the corresponding rational-valued functions, and let

$$
f(x)=\sum_{\nu=1}^{\infty}\left(r_{\nu}(x)\right)^{\nu} \quad\left(x \in G_{1}\right) .
$$


If $h$ is an arbitrary fixed element of $G_{1}$, there is an integer $N=N(h)$ such that $\nu>N$ implies $r_{\nu}(h)=0$. Thus, from the additivity of the $r_{\nu}$,

$$
f(x+h)-f(x)=\sum_{\nu=1}^{N}\left[\left(r_{\nu}(x)+r_{\nu}(h)\right)^{\nu}-\left(r_{\nu}(x)\right)^{\nu}\right],
$$

a polynomial.

It may be noticed that the function $f$ in Example 1 satisfies (P1). In fact, the following is true: if $G$ is an arbitrary abelian group, and if $P$ is a function on $G$ such that, for each $(a, b) \in G \times G$, the function given by $\Delta_{b} P(a+\lambda b)$ $(\lambda \in C)$ is a polynomial on $C$, then $P$ has property $(\mathrm{P} 1)$. For, given $(a, b)$, it is easy to construct a polynomial $Q_{a, b}$ on $C$ such that

$$
\begin{aligned}
Q_{a, b}(\lambda+1)-Q_{a, b}(\lambda) & =\Delta_{b} P(a+\lambda b) \quad(\lambda \in C), \\
Q_{a, b}(0) & =P(a) .
\end{aligned}
$$

Then $P(a+\lambda b)=Q_{a, b}(\lambda)$.

Example 1 shows that, in general, the degree $N_{a, b}$ of $P_{a b}$ is not a bounded function on $G \times G$. Even on $C+C$, there exists a function satisfying (P1) with $N_{a, b}$ unbounded [4].

Proof of Theorem 1. Sufficiency. Let $f$ be a function on $G$ such that $\Delta_{h} f$ is a polynomial for each $h \in G$. Then $f$ may be taken to be a continuous function, since otherwise there is an additive function $\Gamma_{1}$ on $G$ such that $f-\Gamma_{1}$ is continuous, and the differences of $f-\Gamma_{1}$ will be polynomials. Also, $f$ has property (P1) and therefore can (and will) be considered simply as a function on $G / B$.

Clearly, $G / B$ contains a dense subgroup $G^{\prime}$ which has a finite Hamel basis, viz., $G^{\prime}=R^{m}+G_{1}$, where $R$ denotes the subgroup of $E$ consisting of the rational numbers. If $H=\left\{h_{1}, \cdots, h_{p}\right\}$ is a Hamel basis for $G^{\prime}$, and if $G^{\prime \prime}$ is the group generated by $H$, then $G^{\prime \prime}$ is isomorphic to $C^{p}$. The isomorphism $\phi$ can be chosen so that $\phi h_{i}=\epsilon_{i}$ has $\delta_{i j}$ as its $j$ th coordinate. Let polynomials $f_{i}(i=1, \cdots, p)$ be defined on $C^{p}$ by

$$
f_{i}(\phi x)=\Delta_{h_{i}} f(x) \quad\left(x \in G^{\prime \prime}\right) .
$$

Then, clearly,

$$
\Delta_{\epsilon_{i}} f_{j}=\Delta_{\epsilon_{j}} f_{i} \quad(i, j=1, \cdots, p) .
$$

But (2.2) implies that there exists a polynomial $g^{*}$ on $C^{p}$ such that $g^{*}\left(x+\epsilon_{i}\right)$ $-g^{*}(x)=f_{i}(x) \quad(i=1, \cdots, p)$. Explicitly, $g^{*}=g_{p}$, where $g_{k}$ is given on $C^{k}$ $(k=1,2, \cdots)$ by

$$
\begin{aligned}
g_{0} \equiv & 0 \\
g_{k}\left(n_{1}, \cdots, n_{k-1}, n_{k}\right)= & g_{k-1}\left(n_{1}, \cdots, n_{k-1}\right) \\
& +\sum_{m=0}^{M} c_{m, k}\left(n_{1}, \cdots, n_{k-1}\right)\left(B_{m+1}\left(n_{k}\right)-B_{m+1}\right) /(m+1) .
\end{aligned}
$$


Here, the $c_{m, k}$ are the polynomials obtained from

$$
f_{k}\left(n_{1}, \cdots, n_{k-1}, n_{k}, 0, \cdots, 0\right)=\sum_{m=0}^{M} c_{m, k}\left(n_{1}, \cdots, n_{k-1}\right)\left(n_{k}\right)^{m},
$$

while $B_{m}(x)$ and $B_{m}$ are the $m$ th Bernoulli polynomial and number respectively. This assertion can be proved by induction on $p$. (Note that the well-known identity

$$
\left(B_{m+1}(x+1)-B_{m+1}(x)\right) /(m+1)=x^{m}
$$

[10] implies that the sum (2.3) is

$$
\left(-\sum_{j=n_{k}}^{-1}+\sum_{j=0}^{n_{k}-1}\right) f_{k}\left(n_{1}, \cdots, n_{k-1}, j\right),
$$

the first (respectively, second) sum in (2.4) being empty if $n_{k} \geqq 0$ (resp., if $\left.n_{k} \leqq 0\right)$.)

Also, $g^{*}$ has a unique extension as a polynomial $g^{* *}$ on all of $R^{p}$. Let $g$ denote the function on $G^{\prime}$ given as follows: if

$$
x \sim \sum_{i=1}^{p} r_{i} h_{i}, \text { then } g(x)=g^{* *}\left(r_{1}, \cdots, r_{p}\right)
$$

Clearly, $g$ satisfies (P1) and is of bounded degree. Since $\Delta_{h_{i}}(f-g)(x)=0$ for each $x \in G^{\prime \prime}(i=1, \cdots, p)$, it follows that $f-g$ is constant on $G^{\prime \prime}$. If $x$ is any point in $G^{\prime}$, then there exists a positive integer $K$ such that $\mu K x \in G^{\prime \prime}$ for all $\mu$ in $C$. Then the polynomial in $\lambda$ given by $f(\lambda x)-g(\lambda x)$, being constant for $\lambda=\mu K(\mu \in C)$, is a constant on $C$, so that $f$ is a polynomial on $G^{\prime}$. If $N-1$ is its degree, then $\Delta_{b}^{N} f(a)$ vanishes for all $(a, b) \in G^{\prime} \times G^{\prime}$, and therefore, by continuity, for all $(a, b) \in G \times G$. But this implies that $f$ is a polynomial of degree $N-1$ on $G$ :

$$
f(a+\lambda b)=\sum_{\mu=0}^{N-1} \sum_{\nu=0}^{\mu}(-1)^{\nu}\left(\begin{array}{l}
\lambda \\
\mu
\end{array}\right)\left(\begin{array}{l}
\mu \\
\nu
\end{array}\right) f(a+\nu b) \quad(\lambda \in C ; a, b \in G) .
$$

3. The difference property for exponential polynomials. A function $z$ on $G$ is a generalized character if $z$ is a continuous homomorphism from $G$ to the multiplicative group of nonzero complex numbers. A function $e$ on $G$ is an exponential polynomial if

$$
e=\sum_{i=1}^{n} P_{i} z_{i}
$$

where each $P_{i}$ is a polynomial and each $z_{i}$ is a generalized character. If each $P_{i}$ is a constant, and each $z_{i}$ is an ordinary character, then $e$ is said to be a trigonometric polynomial. 
Theorem 2. Let $G$ be an abelian locally compact group. A necessary and sufficient condition in order that the class of exponential polynomials on $G$ have the difference property is that $G$ be compactly generated.

Let $f$ be a function on $G$ such that

$$
\Delta_{h} f=\sum_{\alpha \in A} P_{h}^{\alpha} z_{\alpha} \quad(h \in G)
$$

where $\left\{z_{\alpha}: \alpha \in A\right\}$ is the set of all generalized characters on $G$, each $P_{h}^{\alpha}$ is a polynomial on $G$, and, for each fixed $h \in G$,

$$
P_{h}^{\alpha}=0 \quad \text { for } \alpha \neq \alpha_{1}(h), \cdots, \alpha_{k(h)}(h) .
$$

Since the class of continuous functions on $G$ has the difference property, $f$ may be taken to be continuous.

Distinct generalized characters are linearly independent over the ring of polynomials on $G$ (Lemma 3.1, below). Hence, $f$ will be an exponential polynomial if and only if there exist polynomials $\left\{Q^{\alpha}: \alpha \in A\right\}$ such that

$$
\Delta_{h}\left(Q^{\alpha} z_{\alpha}\right)=P_{h}^{\alpha} z_{\alpha} \quad(h \in G, \alpha \in A),
$$

and

$$
Q^{\alpha}=0 \quad \text { for } \alpha \neq \alpha_{1}, \cdots, \alpha_{k},
$$

for then it is clear that

$$
f=\sum_{\alpha \in A} Q^{\alpha} z_{\alpha} .
$$

The proof of the sufficiency portion of Theorem 3 consists in constructing polynomials $Q^{\alpha}$ satisfying (3.3), and showing that (3.4) also holds. If $G$ is not compactly generated, however, then it is not true that (3.2) and (3.3) imply (3.4), even when $\left\{\Delta_{h} f: h \in G\right\}$ are all trigonometric polynomials. This is shown in Theorem 3 , from which the necessity of the condition in Theorem 2 will follow.

Lemma 3.1. Let $G$ be an arbitrary group, let $z_{1}, \cdots, z_{n}$ be distinct homomorphisms of $G$ into the multiplicative group of nonzero complex numbers, and let $P_{1}, \ldots, P_{n}$ be complex functions satisfying (P1). If

$$
P_{1} z_{1}+\cdots+P_{n} z_{n} \equiv 0,
$$

then $P_{1} \equiv \cdots \equiv P_{n} \equiv 0$.

Proof. First, consider the special case $G=C$. Since $z_{1}, \cdots, z_{n}$ are distinct, the complex numbers $z_{1}(1), \cdots, z_{n}(1)$ are necessarily distinct. If not all $P_{j}$ are identically zero, then, reordering if necessary, it may be assumed that for some integer $p, 1 \leqq p \leqq n$, 


$$
\begin{aligned}
z_{j}(1) / z_{1}(1) & =e^{i \beta_{j}} \neq 1 \quad\left(\beta_{j} \text { real, } 2 \leqq j \leqq p\right), \\
\left|z_{1}(1)\right| & >\left|z_{j}(1)\right| \quad(p+1 \leqq j \leqq n), \\
P_{j}(\lambda) & =c_{j} \lambda^{m}+O\left(\lambda^{m-1}\right) \text { as } \lambda \rightarrow \infty \quad\left(1 \leqq j \leqq p ; c_{1} \neq 0\right) .
\end{aligned}
$$

It follows upon division of the terms of (3.5) by $\lambda^{m} z_{1}(\lambda)$ that

$$
c_{1}=-\sum_{j=2}^{p} c_{j} e^{i \beta_{j \lambda}}+O\left(\lambda^{-1}\right) \text { as } \lambda \rightarrow \infty .
$$

Thus, taking $\lambda=1,2, \cdots, N$ in (3.6), adding, and dividing by $N$, it is seen that

$$
c_{1}=-(1 / N) \sum_{j=2}^{p} \frac{c_{j} e^{i \beta_{j}}\left(e^{i \beta_{j} N}-1\right)}{e^{i \beta_{j}}-1}+O\left(N^{-1} \log N\right) .
$$

Letting $N$ approach infinity in (3.7), it follows that $c_{1}=0$, a contradiction. In the general case, we prove by induction on $n$ that $P_{1}(x) \equiv 0$. This is obvious for $n=1$, since $z_{1}$ is never zero. Now let $n>1$, and suppose the result holds for all $p<n$. Choose $x_{0} \in G$ such that $z_{n}\left(x_{0}\right) \neq z_{1}\left(x_{0}\right)$; this is possible since the $z$ 's are distinct. Suppose that $z_{1}\left(x_{0}\right)=z_{2}\left(x_{0}\right)=\cdots=z_{p}\left(x_{0}\right)$ for some integer $p, 1 \leqq p<n$, while $z_{j}\left(x_{0}\right) \neq z_{1}\left(x_{0}\right)$ for $p<j \leqq n$. Then

$$
\begin{aligned}
\sum_{j=1}^{n} P_{j}\left(x+\lambda x_{0}\right) z_{j}\left(x+\lambda x_{0}\right)= & {\left[\sum_{j=1}^{p} P_{j}\left(x+\lambda x_{0}\right) z_{j}(x)\right] z_{1}\left(\lambda x_{0}\right) } \\
& +\sum_{j=p+1}^{n} P_{j}\left(x+\lambda x_{0}\right) z_{j}\left(x+\lambda x_{0}\right) .
\end{aligned}
$$

For each fixed $x$, this expression can be considered as an exponential polynomial on $C$, and $z_{1}^{\prime}(\lambda)=z_{1}\left(\lambda x_{0}\right)$ is distinct from the other generalized characters. Its coefficient is therefore zero for each $\lambda \in C$. Hence, for each $x \in G$

$$
\sum_{j=1}^{p} P_{j}(x) z_{j}(x)=0
$$

so that $P_{1}(x) \equiv 0$, by the induction assumption.

LEмmA 3.2. Let $G$ be an abelian topological group, let $z \neq \equiv 1$ be a generalized character on $G$, and let $\left\{P_{h}: h \in G\right\}$ be a collection of polynomials on $G$ such that

$$
P_{h}\left(x+h^{\prime}\right) z\left(h^{\prime}\right)-P_{h}(x)=P_{h^{\prime}}(x+h) z(h)-P_{h^{\prime}}(x),
$$

for all $h, h^{\prime}, x$ in $G$. Then there exists a polynomial $Q$ on $G$ such that

$$
\Delta_{h}(Q z)=P_{h} z \quad(h \in G) .
$$

Proof. Let $h \in G$ be such that $|z(h)|<1$, or, if there is no $h$ with this property, i.e., if $|z| \equiv 1$, let $z(h) \neq 1$. Consider the expression 


$$
\lim _{r \rightarrow 1-}\left\{-\sum_{n=0}^{\infty}(z(h) r)^{n} P_{h}(x+n h)\right\} \quad(x \in G) .
$$

If $|z(h)|<1$, then the $r$ in the summation may be replaced by 1 , and the lim omitted. Since $P_{h}$ is a polynomial,

$$
P_{h}(x+n h)=\sum_{k=0}^{N} c_{k}(x) n^{k} \quad(x \in G)
$$

where each $c_{k}(x)$ is a polynomial on $G$, obtainable explicitly from setting $n=0,1, \cdots, N$ in (3.11) and solving by Cramer's rule. The sum in (3.10) is given for $0<r<1$ by

$$
-\sum_{k=0}^{N} c_{k}(x) \sum_{n=0}^{\infty} n^{k}(z(h) r)^{n}=-\left.\sum_{k=0}^{N} c_{k}(x)\left\{y \frac{d}{d y}\right\}^{k}\left(\frac{1}{1-y}\right)\right|_{y=r z(h)} .
$$

Hence the limit in (3.10) exists and yields a polynomial on $G$; let this polynomial be denoted by $Q$. Let $h^{\prime}$ and $x$ be arbitrary elements of $G$. Then

$$
Q\left(x+h^{\prime}\right) z\left(h^{\prime}\right)-Q(x)
$$

$$
=\lim _{r \rightarrow 1-}\left\{-\sum_{n=0}^{\infty}(z(h) r)^{n}\left[P_{h}\left(x+h^{\prime}+n h\right) z\left(h^{\prime}\right)-P_{h}(x+n h)\right]\right\} .
$$

From (3.8), the right-hand side of $(3.12)$ is

$$
\begin{aligned}
\lim _{r \rightarrow 1-}\{ & \left.-\sum_{n=0}^{\infty}(z(h) r)^{n}\left[P_{h^{\prime}}(x+(n+1) h) z(h)-P_{h^{\prime}}(x+n h)\right]\right\} \\
= & P_{h^{\prime}}(x)-\lim _{r \rightarrow 1-}(1-r) \sum_{n=1}^{\infty} P_{h^{\prime}}(x+n h) z(h)^{n} r^{n-1}=P_{h^{\prime}}(x),
\end{aligned}
$$

so that (3.9) follows from (3.12) and (3.13).

Proof of Theorem 2. Sufficiency. Let $f$ be a function (which may and will be assumed to be continuous) such that (3.1) and (3.2) hold. From (3.1), Lemma 3.1, and the identity

$$
\Delta_{h^{\prime}} \Delta_{h} f=\Delta_{h} \Delta_{h^{\prime}} f,
$$

it follows that (3.8) holds for each generalized character $z$. For each $z_{\alpha}$ except $z_{0} \equiv 1$, it follows from Lemma 3.2 that there exists a polynomial $Q^{\alpha}$ satisfying (3.9); in particular, if $P_{h}^{\alpha} \equiv 0$ for all $h$, then $Q^{\alpha} \equiv 0$. But only finitely many $Q^{\alpha}$ can be nonzero. For suppose that $Q^{i} \not \equiv 0 \quad(i=1,2, \cdots)$, with $P_{h}^{i}$ and $z_{i}$ the corresponding polynomials and generalized characters. For each $h \in G$, (3.2) shows that there exists an integer $i(h)$ such that $P_{h}^{i} \equiv 0$ for $i \geqq i(h)$. Hence, from (3.3),

$$
Q^{i}(x+h) z_{i}(h)-Q^{i}(x) \equiv 0 \quad(i \geqq i(h), x \in G),
$$


whence it follows that $z_{i}(h)=1$ for $i \geqq i(h)$. Let

$$
H_{j}=\left\{h: h \in G, z_{i}(h)=1 \text { for all } i \geqq j\right\} .
$$

$H_{j}$ is a closed subgroup of $G, H_{j+1} \supset H_{j}$ and $G=\bigcup H_{j}$, so that at least one $H_{j}$ is of positive Haar measure, and thus open [5]. Therefore $H_{k}$ is open for all $k \geqq j$. Let $A$ be a compact neighborhood of 0 which generates $G$. Then $\bigcup\left\{H_{k}: k \geqq j\right\}$ covers $G$, so that $A$ is covered by some $H_{N}$, whence $H_{N}=G$. Therefore $z_{i} \equiv 1$ for all $i \geqq N$, contradicting the distinctness of the $z_{i}$. Since $Q^{\alpha} \equiv 0$ for all but finitely many $\alpha$, the function given by $g=\sum Q^{\alpha} z_{\alpha}$ (the summation taken for all $\alpha$ such that $z_{\alpha} \neq 1$ ) is an exponential polynomial, and, for each $h, \Delta_{h}(f-g)$ is clearly a polynomial on $G$. But the function $f-g$ is continuous, and the class of polynomials on $G$ has the difference property from Theorem 1 , since $G$ is compactly generated only if $G_{1}$ is finitely generated. Hence, $f-g$ is a polynomial on $G$.

In the proof just given, use was made of the fact that a compactly generated group $G$ is not the countable union of a strictly increasing sequence of closed subgroups. Conversely,

LEMMA 3.3. If the locally compact abelian group $G$ is not compactly generated, there is a sequence $\left\{H_{j}\right\}$ of closed subgroups of $G$, such that $H_{j} \subset H_{j+1}$ (strictly) and $\bigcup H_{j}=G$.

Proof. There is a compact subgroup $G^{\prime}$ of $G$ such that $G / G^{\prime}=E^{p}+G_{2}$, with $G_{2}$ discrete. Since $G$ is not compactly generated, it follows that $G_{2}$ is not finitely generated. It is known [9] that

$$
G_{2}=\bigcup_{n=1}^{\infty} S_{n},
$$

where each $S_{n}$ is a direct sum of cyclic groups, and $S_{n} \subset S_{n+1}$. If the inclusion is proper for infinitely many $n$, the choice of the $H_{j}$ is clear, and the lemma follows. Otherwise, $G_{2}$ is itself a direct sum of infinitely many cyclic groups:

$$
G_{2}=\sum_{\alpha} A_{\alpha} \text {. }
$$

Let $\left\{A_{\alpha_{1}}, A_{\alpha_{2}}, \ldots\right\}$ be a countably infinite subset of $\left\{A_{\alpha}\right\}$, and let

$$
H_{j}=E^{p}+\sum\left\{A_{\alpha}: \alpha \neq \alpha_{j+1}, \alpha_{j+2}, \cdots\right\} \text {. }
$$

Then $H_{j} \subset H_{j+1}$ properly, and their union is $G$.

Theorem 3. Let $G$ be an abelian locally compact group. The class of trigonometric polynomials on $G$ has the difference property if and only if $G$ is compactly generated.

Proof. The sufficiency is clearly a corollary of the sufficiency proof of Theorem 2 . If $G$ is not compactly generated, let $\left\{H_{j}\right\}$ be the sequence given 
by Lemma 3.3 , and for each $j$ let $z_{j}$ be a character identically 1 on $H_{j}$ but not identically 1 on $G$; such characters exist [11]. Let $\sum a_{j}$ be a convergent infinite series of positive numbers, and let $f$ be defined by

$$
f=\sum_{j=1}^{\infty} a_{j} z_{j} .
$$

If $h \in G$ is given, there exists an integer $k=k(h)$ such that $h \in H_{k+1}$. Then

$$
f(x+h)-f(x)=\sum_{j=1}^{k} a_{j}\left(z_{j}(h)-1\right) z_{j}(x),
$$

a trigonometric polynomial.

Suppose now that $f$, given by (3.14) is also given by

$$
f=\sum_{i=1}^{n} P_{i} z_{\alpha_{i}}+\Gamma,
$$

with polynomials $P_{i}$, generalized characters $z_{\alpha_{i}}$, and an additive function $\Gamma$. Let $z_{j}$ be a character appearing in (3.14) but not in (3.15), and let $h \in G$ be chosen such that $z_{j}(h) \neq 1$. Then $z_{j}$ appears in the expression for the exponential polynomial $\Delta_{h} f$ obtained from (3.14) but not in that obtained from (3.15). This contradicts Lemma 3.1. Thus the necessity portions of both Theorem 2 and of Theorem 3 are established.

\section{REFERENCES}

1. N. G. de Bruijn, Functions whose differences belong to a given class, Nieuw Arch. Wisk. 23 (1951), 194-218.

2.,$A$ difference property for Riemann integrable functions and for some similar classes of functions, Nederl. Akad. Wetensch. Proc. Ser. A 55 (1952), 145-151.

3. F. W. Carroll, Difference properties for continuity and Riemann integrability on a locally compact group, Trans. Amer. Math. Soc. 102(1962), 284-292.

4.

5. P. R. Halmo, Measure theory, Van Nostrand, New York, 1950, p. 266.

6. E. Hille and R. S. Phillips, Functional analysis and semi-groups, Amer. Math. Soc. Colloq. Publ. Vol. 31, Amer. Math. Soc., Providence, R. I., 1957, p. 760.

7. J. H. B. Kemperman, A general functional equation, Trans. Amer. Math. Soc. 86 (1957), 28-56.

8. - On exponential polynomials, unpublished manuscript.

9. A. G. Kurosh, The theory of groups, Vol. 1, Chelsea, New York, 1955, p. 175.

10. N. E. Nörlund, Differenzenrechnung, New York, 1954, p. 19.

11. L. S. Pontrjagin, Topologische Gruppen, Vol. 2, Teubner, Leipzig, 1958, pp. 47-50.

12. A. Weil, L'integration dans les groupes topologiques et ses applications, Hermann, Paris, 1953, p. 110.

Ohio State University,

Columbus, Ohio 